\section{Gleichgewichtssinn bei Migräne: Je schwerer die Erkrankung desto schlechter die Balance}

\author{
Migräneattacken hinterlassen Spuren im Gehirn. Das hat wohl auch \\ Auswirkungen auf das Gleichgewichtsorgan. Eine monozentrische Studie \\ korreliert erstmals das Ausmaß von Balance-Störungen mit dem Schwere- \\ grad der Migräne.
}

$P_{\text {tata }}^{a}$ atienten mit Migräne haben nicht selten mehr oder weniger stark ausgeprägte Schwierigkeiten mit der Balancekontrolle. Denkbar ist eine pathophysiologische Verbindung zwischen Migräneattacken und (ischämisch bedingten) Läsionen im posterioren Hirnstromgebiet und einer Vestibularisstörung. Demzufolge müssten Patienten mit häufigen Attacken sowie begleitender Aura stärkere Gleichgewichtsstörungen haben als ihre nicht so stark betroffenen Leidensgenossen. Eine monozentrische Studie untersuchte diese Hypothese.

Einbezogen waren 105 Frauen im Alter zwischen 18 und 55 mit einer ICHDIII-diagnostizierten Migräne mit und ohne Aura sowie mit chronischer Migräne zusammen mit 35 gesunden Kontrollprobandinnen. Mögliche Störungen der Gleichgewichtskontrolle wurden mithilfe des modifizierten Sensory Organization-Tests (mSOT) und des Limits of Stability-Tests (LoS) untersucht. Beim mSOT steht der Proband in standardisierter Position auf einer vibrierenden Kraftmessplatte, die während standardisierter Abläufe - zum Beispiel mit geöff-

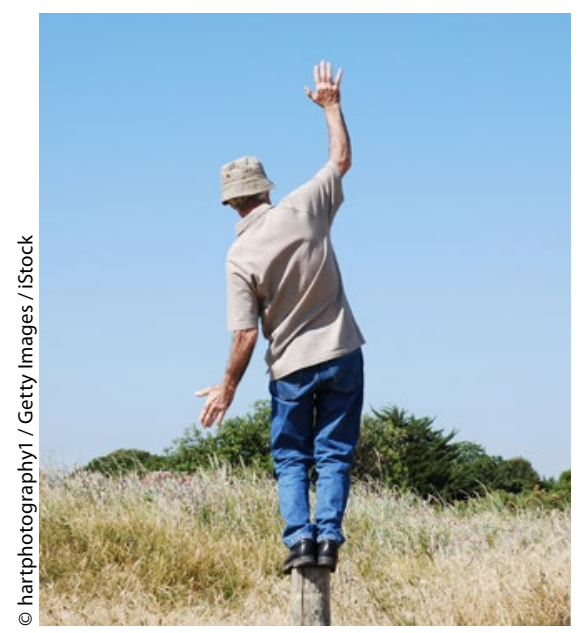

Für Patienten mit Migräne wohl eine der schwierigeren Übungen.

neten und geschlossenen Augen - die Belastungsausschläge misst. Beim LoS werden verschiedene Parameter bei im festen Stand ausgeführten Körperneigungen in verschiedene Richtungen erfasst. Die Beurteilung der Messergebnis- se erfolgte durch einen neutralen Beobachter, der über den Erkrankungsstatus des Teilnehmers nicht informiert war.

Insgesamt zeigten Probanden mit einer Aura-Migräne und einer chronischen Migräne im mSOT signifikant schlechtere Ergebnisse in der Balancekontrolle als Probanden ohne Migräne. In den erschwerten mSOT-Versuchen mit einer schaumstoffbeschichteten Platte schnitten Patienten mit Aura und chronischer Migräne ebenfalls signifikant schlechter ab als Probanden ohne Aura. Im LoS ergaben sich signifikante Unterschiede zwischen allen Migränegruppen und der Kontrollgruppe bei der Reaktionszeit, der Bewegungsgeschwindigkeit und der maximalen Körperauslenkung. Erfasste Kovariablen wie BMI, Medikamenteneinnahme, körperliches Training oder Vorhandensein von iktalem oder interiktalem Schwindel beeinflussten die Ergebnisse nicht.

Fazit: Migräne-Patienten haben einen eingeschränkten Gleichgewichtssinn beziehungsweise eine schlechtere BalanceFähigkeit als gesunde Probanden. Die Gleichgewichtstörungen sind vor allem bei Patienten mit Aura und mit chronischer Migräne ausgeprägt. Betroffenen mit klinisch relevanten Störungen sollte ein spezifisches Balance-Training angeboten werden. Dr. Barbara Kreutzkamp

Carvalho GF et al. Balance impairments in different subgroups of patients with migraine. Headache 2017; 57:363-74

\section{CFS bei Jugendlichen: Fast jeder Sechste ist auch chronisch schmerzgeplagt}

Das chronische Müdigkeitssyndrom (CFS) und chronische Schmerzsyndrome überlappen sich. Ob das schon bei Jugendlichen zutrifft, sollte eine Geburtskohortenstudie zeigen. Denn zusätzliche Schmerzen erfordern eine komplexere CFS-Therapie.

$D$ a atienten mit chronischem Müdigkeitssyndrom (chronic fatigue syndrome, CFS) klagen oftmals auch über Schmerzen, was sich unter anderem in den CFSDiagnoseschlüsseln widerspiegelt. Die Überlappung von CFS mit CWP (chroni- sche Schmerzen in mehreren Körperregionen, chronic widespread pain) bei Erwachsenen ist bekannt. Im Rahmen der Avon Longitudinal Study of Parents and Children (ALSPAC) sollten nun auch mögliche Assoziation bei 17-jährigen Ju- gendlichen näher untersucht werden nicht zuletzt, um in der CFS-Therapie die Schmerzkomponente besser als bisher berücksichtigen zu können.

Ausgewertet wurden dazu Schmerzfragebögen und strukturierte klinische Interviews von 3.215 in 1991/92 geborenen Teilnehmern der ALSPAC-Kohorte. Anhand der Angaben aus den Schmerzfragebögen konnte bei 4,5\% der Jugendlichen auf ein CWP und anhand der Angaben im klinischen Interview bei 2,6\% auf ein CFS geschlossen werden. Jugendliche mit einem CFS hatten eine deutlich gesteigerte Wahrscheinlichkeit für ein zusätzliches CWP (Odds ratio 3,87; 95\%- 